\title{
Changes in Blood Pressure and Heart Rate during Decompressive Craniectomy
}

\author{
Kwang Wook Jo, ${ }^{1}$ Hyun-Ju Jung, ${ }^{2}$ Do Sung Yoo, ${ }^{3}$ Hae-Kwan Park ${ }^{3}$ \\ Department of Neurosurgery, Bucheon St. Mary's Hospital, College of Medicine, The Catholic University of Korea, Seoul, Korea \\ Department of Anesthesiology, ${ }^{2}$ Uijeongbu St. Mary's Hospital, College of Medicine, The Catholic University of Korea, Seoul, Korea \\ Department of Neurosurgery, ${ }^{3}$ Eunpyeong St. Mary's Hospital, College of Medicine, The Catholic University of Korea, Seoul, Korea
}

Objective : Rapid increase in intracranial pressure (ICP) can result in hypertension, bradycardia and apnea, referred to as the Cushing phenomenon. During decompressive craniectomy (DC), rapid ICP decreases can cause changes in mean atrial blood pressure (mABP) and heart rate (HR), which may be an indicator of intact autoregulation and vasomotor reflex.

Methods : A total of 82 patients who underwent DC due to traumatic brain injury (42 cases), hypertensive intracerebral hematoma (19 cases), or major infarction (21 cases) were included in this prospective study. Simultaneous ICP, mABP, and HR changes were monitored in one minute intervals during, prior to and 5-10 minutes following the DC.

Results : After DC, the ICP decreased from $38.1 \pm 16.3 \mathrm{mmHg}$ to $9.5 \pm 14.2 \mathrm{mmHg}(p<0.001)$ and the mABP decreased from $86.4 \pm 14.5$ $\mathrm{mmHg}$ to $72.5 \pm 11.4 \mathrm{mmHg}(p<0.001)$. Conversly, overall HR was no significantly changed in HR, which was $100.1 \pm 19.7 \mathrm{rate} / \mathrm{min}$ prior to $D C$ and $99.7 \pm 18.2$ rate/min ( $p=0.848)$ after $D C$. Notably when the HR increased after $D C$, it correlated with a favorable outcome $(p<0.001)$, however mortality was increased $(p=0.032)$ when the HR decreased or remained unchanged.

Conclusion : In this study, ICP was decreased in all patients after DC. Changes in HR were an indicator of preserved autoregulation and vasomotor reflex. The clinical outcome was improved in patients with increased HR after DC.

Key Words : Prognosis · Decompressive craniectomy · Heart rate $\cdot$ Intracranial pressure $\cdot$ Mean arterial blood pressure.

\section{INTRODUCTION}

Despite highly developed medical and neurosurgical treatments, the mortality and morbidity of patients with intractable increased intracranial pressure (ICP) remains challenging $^{3,14,19,36,37)}$. Prior studies have reported both benefits and limitations associated with decompressive craniectomy (DC) in patients with hypertensive intracerebral hemorrhage $(\mathrm{H}-$
$\mathrm{ICH})^{4,17)}$, traumatic brain injury $(\mathrm{TBI})^{3,14,24,36)}$, and major infarction $(\mathrm{MI})^{12,16,24,27,36,37)}$. Often DC is the last choice of treatment, though, it remains the most effective treatment for patients with uncontrollable brain swelling. The surgical procedures for DC include removing the bone over large areas of the calvarium, converting a "closed box" with a finite volume into an open one. It is a rapid and effective surgical method for uncontrollable increase in ICP. After DC, surgery the ICP

- Received : December 21, 2020 •Revised : February 3, 2021 •Accepted : April 28, 2021

- Address for reprints : Do Sung Yoo

Department of Neurosurgery, Eunpyeong St. Mary's Hospital, College of Medicine, The Catholic University of Korea, 1021 Tongil-ro, Eunpyeong-gu, Seoul 03312, Korea Tel : +82-2-2030-4491, Fax : +82-2-2030-4626, E-mail : yooman@catholic.ac.kr, ORCID : https://orcid.org/0000-0003-2569-6502

This is an Open Access article distributed under the terms of the Creative Commons Attribution Non-Commercial License (http://creativecommons.org/licenses/by-nc/4.0) which permits unrestricted non-commercial use, distribution, and reproduction in any medium, provided the original work is properly cited. 
will be decreased, and cerebral perfusion pressure can be improved to an adequate level. Moreover, DC will allow an edematous brain to herniate through the craniectomy opening rather than through the tentorial incisura, thereby avoiding brain stem compression ${ }^{5,36,37)}$.

Rapidly increasing ICP can trigger the Cushing reflex which cause hypertension, bradycardia, and respiratory irregularities, all of which are signs of subsequent brain herniation and death ${ }^{1,6,11,18,31,38)}$. In situations where there is acute elevation of ICP, the Cushing reflex occurs. Decreasing the ICP may be critical to improving clinical outcomes and saving the patient's life. Several pathophysiological studies refined Cushing's findings by showing an initial tachycardia associated with hypertension followed by bradycardia. The initial hypertensive and tachycardia symptoms were due to the elevated activity of the sympathetic nervous system and late bradycardia was mediated by parasympathetic nerve activation ${ }^{1,6,7,10,11,20-23,29,31)}$.

In the current study, it was hypothesized that the Cushing's reflex could be an indicator of intact autoregulation. Under this hypothesis, increased heart rate (HR) compensated for decreased mean atrial blood pressure (mABP) following DC may reflect an intact vasomotor reflex. Patients with intact autoregulation and vasomotor reflex showed more favorable outcomes compared to patients without these reflexes ${ }^{1,23)}$.

The current results indicate that the mABP was generally decreased during DC, while the ICP decreased rapidly. Thus, changes in the HR were also investigated. If autoregulation and the vasomotor response were intact, the mABP decrease might influence the HR to preserve cerebral perfusion. $\mathrm{Pa}-$ tients with intact autoregulation and vasomotor responses, may have better clinical outcomes than patients without intact responses.

\section{MATERIALS AND METHODS}

This prospective, observational study was approved by the Institutional Review Board of Eunpyeong St. Mary's Hospital. The treatment protocol (Fig. 1) was also approved by the Institutional Review Board of Eunpyeong St. Mary's Hospital (PC17RESI0027). All patients or their representatives provided written consent to treatment.

\section{Patient selection}

Eighty-two patients with massive brain swelling due to TBI (42 cases), H-ICH (19 cases) or MI (21 cases), that were treated by DC with dura expansion between January 2017 and June 2020, were included in the current study. Patients were successfully monitored for ventricular ICP, mABP, and HR changes before and after the DC (Table 1). This study included 82 patients. There were 59 males and 23 females with a mean age of $49.7 \pm 156.4$ years (range, $8-77$; median age, 53 years).

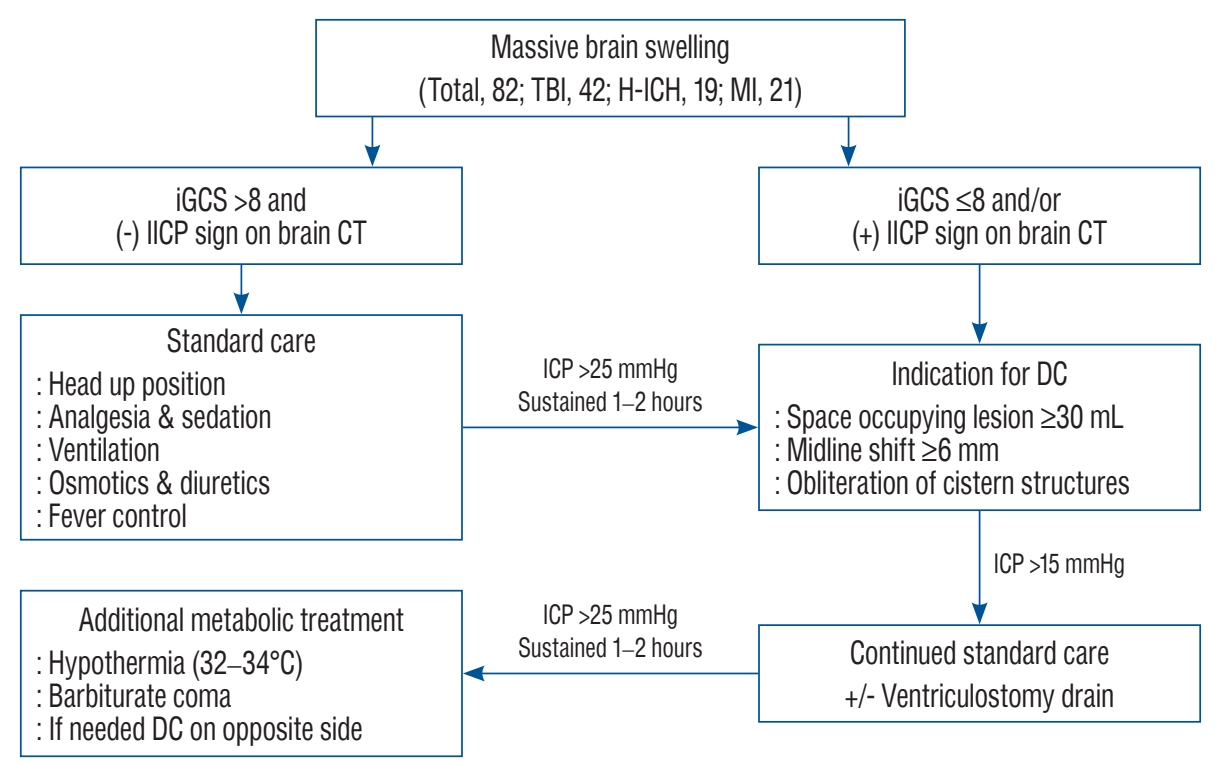

Fig. 1. Flow sheet of treatment protocol. TBI : traumatic brain injury, $\mathrm{H}-\mathrm{ICH}$ : hypertensive intracerebral hemorrhage, $\mathrm{MI}$ : major infarction, iGCS : initial Glasgow coma scale, IICP : increased intracranial pressure, CT : computed tomography, DC : decompressive craniectomy. 
Standard management was performed including computed tomography (CT) scan as rapidly as possible when neurological deterioration was apparent. ICP was monitored during the DC surgery, 58 patients were monitored with a CPP-monitoring device (CPP-monitor; Spiegelberg, Hamburg, Germany) and the remaining 24 with a Philips patient monitor (IntelliVue MX700; Phillips Medizin Systeme Boeblingen GmbH, Böblingen, Germany).

\section{Indications for surgery and postoperative man- agement}

Indications for DC were the appearance of massive uni- or bilateral brain swelling on CT scans, with correlating clinical deterioration; worsening of Glasgow coma scale (GCS) less than 8-9 and/or dilation of the pupils unresponsive to light; a midline shift of more than $6 \mathrm{~mm}$; and/or obliteration of the perimesencephalic cistern on CT scans. None of the patients

Table 1. Characteristics and clinical outcomes of the 82 patients treated with DC

\begin{tabular}{|c|c|c|c|c|c|}
\hline & \multicolumn{3}{|c|}{ Etiology } & \multirow{2}{*}{ Total cases } & \multirow{2}{*}{$p$-value* } \\
\hline & TBI & $\mathrm{H}-\mathrm{ICH}$ & MI & & \\
\hline Patient & 42 & 19 & 21 & 82 & \\
\hline \multicolumn{6}{|l|}{ Age (years) } \\
\hline Mean $\pm S D$ & $48.3 \pm 19.1$ & $50.2 \pm 11.8$ & $52.2 \pm 14.3$ & $49.7 \pm 16.4$ & TBI vs. $\mathrm{H}-\mathrm{ICH}(p=0.015)$ \\
\hline Median & 46 & 55 & 55 & 53 & \\
\hline Range & $8-77$ & $25-65$ & $25-76$ & $8-77$ & \\
\hline Male sex & $37(88.1)$ & $11(57.9)$ & $11(52.4)$ & $59(72.0)$ & \\
\hline \multicolumn{6}{|l|}{ GCS score } \\
\hline Mean $\pm S D$ & $5.5 \pm 2.0$ & $5.8 \pm 1.9$ & $6.0 \pm 1.8$ & $5.7 \pm 1.9$ & \\
\hline Median & 5 & 6 & 6 & 5 & \\
\hline Range & $3-10$ & $3-9$ & $4-10$ & $3-10$ & \\
\hline Initial ICP & $32.8 \pm 12.7$ & $44.6 \pm 16.2$ & $42.2 \pm 19.0$ & $38.1 \pm 16.0$ & TBI vs. MI $(p=0.027)$ \\
\hline Post DC ICP & $10.4 \pm 19.9$ & $7.4 \pm 4.4$ & $10.1 \pm 4.7$ & $9.5 \pm 14.1$ & \\
\hline ICP difference & $22.3 \pm 19.5$ & $37.2 \pm 16.1$ & $32.1 \pm 19.2$ & $28.7 \pm 19.4$ & \\
\hline Initial mABP & $83.1 \pm 11.9$ & $91.7 \pm 16.3$ & $88.0 \pm 16.3$ & $86.4 \pm 14.5$ & \\
\hline Post DC mABP & $70.5 \pm 11.7$ & $75.9 \pm 10.5$ & $73.5 \pm 11.2$ & $72.5 \pm 14.5$ & \\
\hline mABP difference & $12.5 \pm 11.2$ & $15.8 \pm 14.5$ & $14.5 \pm 17.3$ & $13.8 \pm 13.6$ & \\
\hline Initial HR & $101.6 \pm 18.2$ & $97.1 \pm 20.4$ & $99.8 \pm 22.6$ & $100.1 \pm 19.7$ & \\
\hline Post DC HR & $102.1 \pm 20.1$ & $96.6 \pm 15.3$ & $97.7 \pm 16.8$ & $99.7 \pm 18.2$ & \\
\hline HR difference & $0.5 \pm 17.1$ & $-0.5 \pm 20.0$ & $-2.1 \pm 15.0$ & $-0.4 \pm 17.2$ & \\
\hline \multicolumn{6}{|l|}{ Clinical outcomes } \\
\hline \multicolumn{6}{|l|}{ GOS } \\
\hline 5 & 17 & 7 & 4 & 28 & \\
\hline 4 & 4 & 2 & 2 & 8 & \\
\hline Favorable & $21(50.0)$ & $9(47.4)$ & $6(28.6)$ & $36(43.9)$ & 0.005, TBI vs. MI \\
\hline 3 & 1 & 2 & 2 & 5 & \\
\hline 2 & 16 & 2 & 2 & 20 & \\
\hline 1 Death & $4(9.5)$ & $6(31.6)$ & $11(52.4)$ & $21(25.6)$ & \\
\hline Median & 2 & 3 & 6 & 3 & \\
\hline
\end{tabular}

Values are presented as mean \pm standard deviation or number (\%). *Fisher's exact test. SD : standard deviation, DC : decompressive craniectomy, TBI : traumatic brain injury, $\mathrm{H}-\mathrm{ICH}$ : hypertensive intracerebral hemorrhage, MI : major infarction, GCS : Glasgow coma scale, ICP : intracranial pressure, mABP : mean arterial pressure, $\mathrm{HR}$ : heart rate, GOS : Glasgow outcome scale 
displayed Cushing's phenomenon corresponding to increased ICP were apparent. Initial ICP monitored after ventricular puncture was high. Patients with primary fatal brainstem failure, indicated by no spontaneous respiration and a persistent GCS score of 3 and/or bilaterally fixed and dilated pupils, did not undergo surgical decompression. Surgical methods (unilateral or bilateral hemicraniectomy) and DC bone size were decided by the operating surgeon. After DC, conventional medical managements, including hyperosmotic agents, hyperventilation, and extraventricular drainage (EVD), were initiated if the ventricular pressure exceeded $20 \mathrm{mmHg}$ (Fig. 1).

\section{Anesthesia and surgical procedures}

All patients were operated on under endotracheal general anesthesia in the supine position. Upon arrival in the operating room, the patient was connected to standard monitoring devices.

Bispectral index (BIS) electrode was positioned on the patient's mandible, contralateral to the lesion. Propofol and remifentanyl infusion rate was targeted to keep BIS values between 40 and 60. A radial arterial catheter was inserted for the continuous monitoring of arterial blood pressure. Anesthesia was induced and maintained with propofol, remifentanil and cisatracurium. Blood pressure was managed by a bolus or continuous infusion of norepinephrine or phenylephrin, as appropriate.

In all patients, ventricular puncture was performed before DC and ICP was monitored during and after the surgery. The EVD tube (EVD catheter; Yushin Medical, Seoul, Korea) was connected to a continuous monitor (CPP-monitor; Spiegelberg, Hamburg, Germany or IntelliVue MX700; Philips Medizin Systeme Boehblingen GmbH, Böblingen, Germany) via a transducer device (Druckmeß-set; Smiths Industries, Grasbrunn, Germany or Pressure Monitoring Set; Edward Lifesciences, Irvine, CA, USA). A large inverted question mark shaped or bicoronal skin flap was used. The limbs were placed just behind the parietal eminence, extending inferiorly to the zygoma and curving anteriorly to the mid-line. The reference points for the bone flaps were : 1) the burr holes in the pterion of frontal bone; 2) the burr holes at the parietal eminences; and 3) the burr holes in the squamous portions. After the ventricular ICP was stabilized, the burr holes were connected using a pneumatic saw, with subsequent removal of the bone flap. The removed bone size was at least $12 \times 15 \mathrm{~cm}$. The frontal median segment of the bone, which was measured approximately 3 to $4 \mathrm{~cm}$ in width along the sagittal sinus, was saved to avoid damage to the sagittal sinus and to function as a framework for later cranioplasty. An additional bone was removed at the temporal region to the floor of the middle fossa. Immediately after the craniectomy, the dura was opened, within 5-10 minutes. Cortical resection of the brain was not performed. In all patient, artificial dura (Lyoplant; B. Braun MelsungenAG, Melsungen, Germany) was placed underneath the incised dura, and secured with several sutures, to allow the brain to herniate outward in a more controlled manner, and to prevent cortical adhesion. Several pieces of Gelform (Gelfoam Sponge; ACE Surgical Supply, Brockton, MA, USA) were inserted to control postoperative epidural bleeding, and preserve the dissection plane, after cranioplasty. The temporalis muscle and skin flap were reapproximated with sutures ${ }^{19)}$. In general situation, during DC, expected blood loss was from about 250 to $500 \mathrm{~mL}$. The blood loss could be somewhat variable according to the patients but it was not definitely influent on the vital sign changes. Typically, the bone flap was maintained in wet gauze at $-70^{\circ} \mathrm{C}$ until reinsertion, at 6 or 8 weeks after the initial surgery. If the autologous bone was not appropriate for reinsertion, the bone fragment for cranioplasty was made using a $3 \mathrm{D}$ printer.

\section{Data collection}

The initial ICP checked after ventricular tapping was considered to be the highest sustained ventricular pressure. The ventricular pressure obtained after craniectomy and dura opening was considered the DC state ICP and dura opening state ICP, respectively. The ventricular pressure was continuously monitored during the operation. The ICP, mABP and HR changes were monitored every minute, and the data just prior the $\mathrm{DC}$ and the most extreme values measured within 10 minutes after the DC were compared. The HR increase or unchanged vs. decrease before and after DC (Table 2). Clinical outcomes were analyzed by Glasgow outcome scale (GOS) score after 6 months after DC. And GOS 5-4 was defined as favorable outcome, GOS 3-2 was defined as a poor outcome and GOS score of 1 was defined as death.

\section{Statistical analysis}

All data were presented as the mean \pm standard deviation (median value). Comparisons between the data collected be- 
Table 2. Clinical outcomes according to the HR change

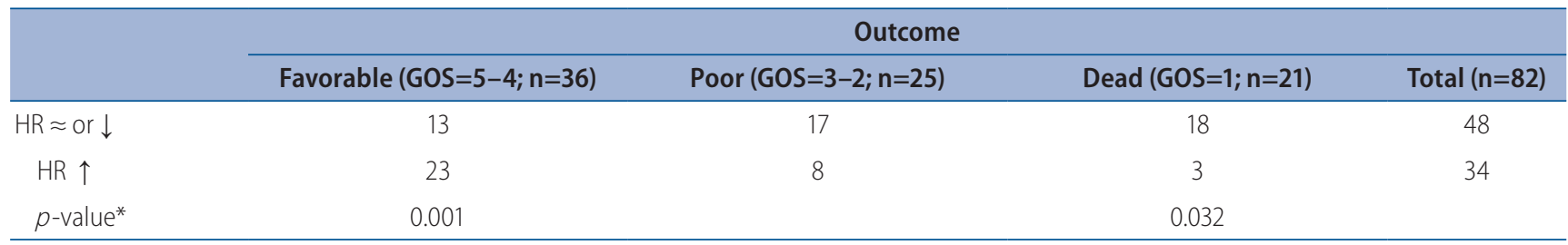

*Fisher's exact test. HR : heart rate, GOS : Glasgow outcome scale

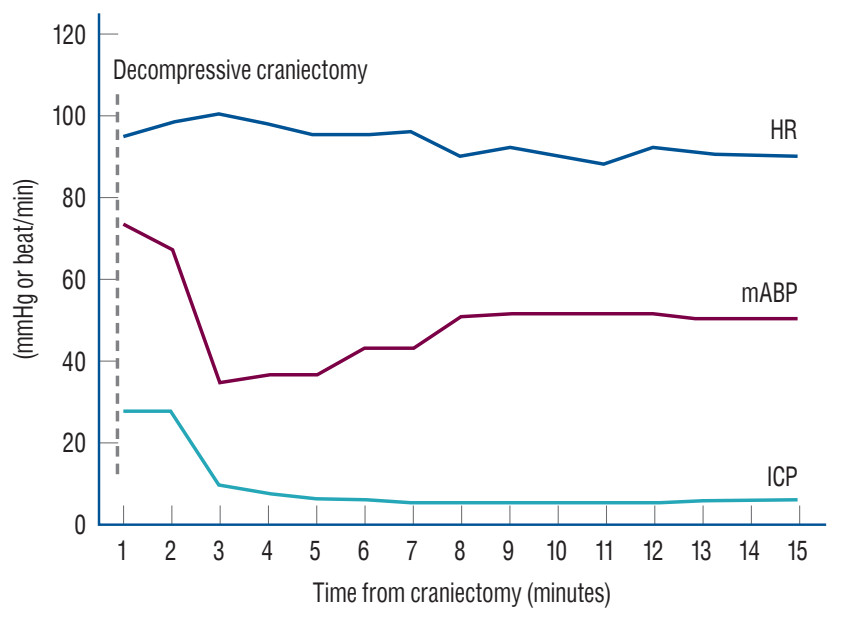

Fig. 2. Demonstration of $I C P, m A B P$, and $H R$ changes during the decompressive craniectomy surgery. $\mathrm{HR}$ : heart rate, $\mathrm{mABP}$ : mean arterial blood pressure, ICP : intracranial pressure.

fore and after surgery were performed using the two-tailed, paired Student's t-test. Differences between categories were compared using a Fisher's exact t-test. Statistical significance set at a value of $p<0.05$. For statistical analysis of the data, nonparametric correlations were determined using SPSS ver. 11.0 (SPSS Inc., Chicago, IL, USA).

\section{RESULTS}

\section{ICP changes during DC surgery}

Before and after DC, the ICP decreased from $38.1 \pm 16.3$ $\mathrm{mmHg}$ to $9.5 \pm 14.2 \mathrm{mmHg}(p<0.001$, Table 1$)$. In all patients, the ICP decreased after DC surgery, and the degree of ICP decrease was correlated with neurologic outcomes $(p=0.013)$.

\section{mABP changes during DC surgery}

On average, the $\mathrm{mABP}$ decreased from $86.4 \pm 14.5 \mathrm{mmHg}$ to $72.5 \pm 11.4 \mathrm{mmHg}(p<0.001)$. In most patients, the mABP was decreased after DC surgery with the exception of eight patients, where the mABP increased after DC surgery. The $\mathrm{mABP}$ changes, before and after DC, were not correlated with clinical outcomes (favorable outcome, $p=0.200$; mortality, $p=0.466$ ).

\section{HR changes during the DC surgery}

The mean HR decreased from $100.1 \pm 19.7$ rate $/ \mathrm{min}$ to $99.7 \pm 18.2 \mathrm{rate} / \mathrm{min}(p=0.848)$ during DC surgery. In $34 \mathrm{pa}-$ tients, the HR was increased, 23 of the 34 patients displayed favorable outcomes. In the remaining 48 patients, the HR was decreased or unchanged, and 18 of those patients were dead. Increased HR after DC was significantly correlated with favorable outcomes $(p<0.001)$. While decreased HR after DC was correlated with mortality ( $p=0.032$, Table 2 ).

\section{Comparisons of statistical results according to the data after DC}

In most patients, systolic blood pressure was decreased by approximately $15 \mathrm{mmHg}$. In some patients, the systolic blood pressure after DC was decreased to below $60 \mathrm{mmHg}$. In these cases, the anesthesiologist typically administered a bolus or continuous infusion of an inotropic agent (norepinephrine or epinephrine). The anesthetic interventions affected $\mathrm{mABP}$ and HR approximately about 5-10 minutes after administration. Data collected during a wide time interval (approximately 30 minute before and after DC), showed quite different values compared with the data with a short range of time interval (data collected before and within 5 minutes after DC) (Fig. 2).

Statistical results of data collected over a wide time interval of about 30 minutes showed no significant difference in favorable outcomes ( $p=0.199)$ or mortality $(p=0.243$ ) (data not shown), but the results of data collected over a short time interval of about 5-10 minutes were significantly correlated with clinical outcomes. When the HR increased after DC, 
there were significant correlations with favorable outcomes $(p<0.001)$, whereas if HR was unchanged or decreased after DC, significantly with mortality ( $p=0.032$, Table 2 ).

\section{DISCUSSION}

Elevated ICP has been found to correlate with adverse outcomes from massive brain swelling. Therefore, accurate knowledge and proper management of ICP are important tools in improving outcomes in patients with massive brain swelling. Many attempts have been made to control massive brain swelling using various medical and surgical treatment modalities, each associated with moderate success ${ }^{13,15,37)}$. Over the past several decades, many studies have shown that DC is an effective treatment strategy in patients with intractable increased ICP caused by a variety of neurosurgical diseas$\mathrm{es}^{3,4,8,12,14,16,17,24,27,28,32,33,35-37)}$.

Hypertension, bradycardia, and respiratory irregularities are the signs of critically increased ICP, which may predict impending brain herniation and death. It is better known as the Cushing reflex, the Cushing reaction, the Cushing phenomenon, the Cushing response, and Cushing's law ${ }^{1,6,11,18,31,38)}$.

Several animal, pathophysiological and clinical observational studies have reported elevated blood pressure in subjects with severely increased ICP. This increase can result from an autoregulation response to maintain cerebral perfusion pressure and cerebral blood flow ${ }^{1,6,10,11,18,20-22,29,31)}$, which may be associated with paroxysmal sympathetic hyperactivi$\mathrm{ty}^{7,10,18,21-23,25)}$. The respiratory irregularity, characterized by Harvey Cushing, is believed to be a phenomenon caused by tonsillar herniation ${ }^{1,6,11,31)}$, which is better known as the Cushing reflex, the Cushing reaction, the Cushing phenomenon, the Cushing response, and Cushing's law ${ }^{1,6,11,18,31,38)}$.

Since the brain is encased in the unyielding vault of the skull, and the brain injury which causes edema and incereased blood flow results in elevated $\mathrm{ICP}^{30)}$. DC surgery is performed to increase the volume of the space available for expanding edematous brain tissue, inducing a shift to the right of the pressure volume curve ${ }^{9,15,24)}$. This results in effectively lowering of the increased ICP, improving cerebral oxygenation and preventing secondary brain damage $\mathrm{e}^{1,12,15,26,34,37)}$.

In the current study, it was hypothesized that the Cushing's reflex could be an indicator of intact autoregulation. Under this hypothesis, HR compensated for decreased mABP following DC may reflect an intact vasomotor reflex. During clinical observation, the initial mABP was not high as we expected in a patient meeting the critieria for the Cushing phenomenon state where the increased ICP could change the $\mathrm{mABP}$ and HR.

It is possible that general anesthesia influences the $\mathrm{mABP}$ in increased ICP patients. Patients with intact autoregulation showed more favorable outcomes compared to patients without it ${ }^{1,23)}$. Once the ICP dropped after DC, the mABP also decreased as the intact autoregulation maintained the cerebral perfusion pressure. The mABP then decreased suddenly and the HR increased as a reflex of the vasomotor response. This phenomenon appears to be the reverse phenomenon of the Cushing reflex ${ }^{23)}$.

A previous study that monitored hemodynamic changes during DC in patients with TBI under general anesthesia found that HR was not changed before and after DC and authors hypothesized that this unchanged HR may have been the result of the data acquisition time, as data were collected before the skin incision and after the hematoma removal ${ }^{2}$. The prior study compared data obtained over a wide range of time before and after DC, however in the current study the data were acquired in a short time range just before and within 5-10 minutes after the DC. This short acquisition time interval was less influenced by the anesthesia that affected the patient's vital signs. If the patient's mABP dropped after DC, the anesthesiologist immediately infused inotropic agents to maintain a stable mABP. Most of the medications given by the anesthesiologist during the operation exerted their pharmacologic effects within several minutes after administration.

In the current study results indicate that the ICP was decreased in all patients and the mABP was decreased in most patients after the DC procedure. However, the HR change was variable among patients, and correlated with clinical outcomes. In patients where the HR decreased after DC surgery, mortality was significantly increased. Conversely, in patients where the HR increased, the chance of a favorable outcome increased as well. A large study using a greater number of patients is needed to determine the robustness and clinical usefulness of this response. Most DC procedures are conducted on critical patients where the anesthetic medications that are used can influence the vital signs.

There were limitations to this study. First, though this was a 
prospective study, but the population may be a homogeneous sample as it was performed in one institution. Second, there was no fixed protocol for anesthesia during DC surgery, therefore the administration of vasoactive medications was not done in a controlled manner, possibly causing bias in statistical analysis. Third, at the beginning of this study, data collection was done by two monitors, one for ICP monitoring device and the other for vital signs monitoring anesthetic machine. Merging data from these two different sources and basing the time measurements on each monitor may have caused bias as well.

\section{CONCLUSION}

ICP and $\mathrm{mABP}$ were decreased in most patients after DC, however the HR changes were variable. The DC procedure was performed in acute critical patients with highly elevated ICP, which was not a result of the Cushing phenomenon. If the HR decreased during DC, the probability of mortality was increased. When HR increased, the probability of a favorable outcome increased. These results suggest that the clinical outcomes differ based on HR changes after DC which may be the result of an intact autoregulation and vasomotor reflex.

\section{CONFLICTS OF INTEREST}

No potential conflict of interest relevant to this article was reported.

\section{INFORMED CONSENT}

Informed consent was obtained from all individual participants included in this study.

\section{AUTHOR CONTRIBUTIONS}

\author{
Conceptualization : KWJ, DSY \\ Data curation : HJJ, HKP \\ Formal analysis : HJJ, HKP \\ Funding acquisition : KWJ, DSY
}

\author{
Methodology : KWJ, DSY \\ Project administration : KWJ, DSY \\ Visualization : HJJ, HKP \\ Writing - original draft : KWJ, HJJ, DSY \\ Writing - review \& editing : KWJ, DSY
}

\section{ORCID}

$$
\begin{array}{ll}
\text { Kwang Wook Jo } & \text { https:/orcid.org/0000-0001-7993-3409 } \\
\text { Hyun-Ju Jung } & \text { https://orcid.org/0000-0001-7258-6758 } \\
\text { Do Sung Yoo } & \text { https://orcid.org/0000-0003-2569-6502 } \\
\text { Hae-Kwan Park } & \text { https://orcid.org/0000-0001-7132-7009 }
\end{array}
$$

\section{- Acknowledgements}

Authors appreciate the help of Neurosurgical nurse specialist (Min-Hee Kim) to collect the data and the grammatical review of our manuscript by Ann C Rice, PhD of the J. Sargeant Reynolds Community College, Richmond, VA, USA.

\section{References}

1. Barbiro-Michaely E, Mayevsky A : Effects of elevated ICP on brain function: can the multiparametric monitoring system detect the 'Cushing Response'? Neurol Res 25 : 42-52, 2003

2. Bharath $\mathrm{S}$, Radhakrishnan M, Umamaheswara Rao GS : Hemodynamic changes during surgical decompression in traumatic brain injury patients. World Neurosurg 136 : e553-e558, 2020

3. Brain Trauma Foundation; American Association of Neurological Surgeons; Congress of Neurological Surgeons; Joint Section on Neurotrauma and Critical Care, AANS/CNS, Bratton SL, et al. : Guidelines for the management of severe traumatic brain injury. VIII. Intracranial pressure thresholds. J Neurotrauma 24 Suppl 1 : S55-S58, 2007

4. Broderick JP, Adams HP Jr, Barsan W, Feinberg W, Feldmann E, Grotta J, et al. : Guidelines for the management of spontaneous intracerebral hemorrhage: a statement for healthcare professionals from a special writing group of the Stroke Council, American Heart Association. Stroke 30 : 905-915,1999

5. Carney N, Totten AM, O'Reilly C, Ullman JS, Hawryluk GW, Bell MJ, et al. : Guidelines for the management of severe traumatic brain injury, fourth edition. Neurosurgery $80: 6-15,2017$

6. Fodstad H, Kelly PJ, Buchfelder M : History of the cushing reflex. Neurosurgery 59 : 1132-1137; discussion 1137, 2006

7. Furuichi S, Endo S, Haji A, Takeda R, Nisijima M, Takaku A : Related changes in sympathetic activity, cerebral blood flow and intracranial 
pressure, and effect of an alpha-blocker in experimental subarachnoid haemorrhage. Acta Neurochir (Wien) 141 : 415-423; discussion 423424, 1999

8. Georgiadis D, Schwarz S, Aschoff A, Schwab S : Hemicraniectomy and moderate hypothermia in patients with severe ischemic stroke. Stroke 33 : 1584-1588, 2002

9. Guerra WK, Gaab MR, Dietz H, Mueller JU, Piek J, Fritsch MJ : Surgical decompression for traumatic brain swelling: indications and results. J Neurosurg 90 : 187-196, 1999

10. Guild SJ, Saxena UA, McBryde FD, Malpas SC, Ramchandra R : Intracranial pressure influences the level of sympathetic tone. Am J Physiol Regul Integr Comp Physiol 315 : R1049-R1053, 2018

11. Heymans $C$ : The control of heart rate consequent to changes in the cephalic blood pressure and in the intracranial pressure. Am J Physiol 85 : 498-506, 1928

12. Hofmeijer J, van der Worp HB, Kappelle LJ : Treatment of space-occupying cerebral infarction. Crit Care Med $31: 617-625,2003$

13. Holtkamp M, Buchheim K, Unterberg A, Hoffmann O, Schielke E, Weber $J R$, et al. : Hemicraniectomy in elderly patients with space occupying media infarction: improved survival but poor functional outcome. J Neurol Neurosurg Psychiatry 70 : 226-228, 2001

14. Hutchinson PJ, Kolias AG, Timofeev IS, Corteen EA, Czosnyka M, Timothy J, et al. : Trial of decompressive craniectomy for traumatic intracranial hypertension. N Engl J Med 375 : 1119-1130, 2016

15. Jiang JY, Xu W, Li WP, Xu WH, Zhang J, Bao YH, et al. : Efficacy of standard trauma craniectomy for refractory intracranial hypertension with severe traumatic brain injury: a multicenter, prospective, randomized controlled study. J Neurotrauma 22 : 623-628, 2005

16. Jüttler $E$, Schwab S, Schmiedek P, Unterberg A, Hennerici M, Woitzik J, et al. : Decompressive surgery for the treatment of malignant infarction of the middle cerebral artery (DESTINY): a randomized, controlled trial.

Stroke 38 : 2518-2525, 2007

17. Juvela S, Heiskanen O, Poranen A, Valtonen S, Kuurne T, Kaste M, et al. : The treatment of spontaneous intracerebral hemorrhage. A prospective randomized trial of surgical and conservative treatment. J Neurosurg $70:$ 755-758, 1989

18. Kalmar AF, Van Aken J, Caemaert J, Mortier EP, Struys MM : Value of cushing reflex as warning sign for brain ischaemia during neuroendoscopy. Br J Anaesth 94 : 791-799, 2005

19. Kim KT, Park JK, Kang SG, Cho KS, Yoo DS, Jang DK, et al. : Comparison of the effect of decompressive craniectomy on different neurosurgical diseases. Acta Neurochir (Wien) $151:$ 21-30, 2009

20. Kocsis $B$, Fedina L, Pasztor E : Effect of preexisting brain ischemia on sympathetic nerve response to intracranial hypertension. J Appl Physiol (1985) $70: 2181-2187,1991$

21. Krasney JA, Koehler RC : Heart rate and rhythm and intracranial pressure. Am J Physiol 230 : 1695-1700, 1976

22. Matsuura S, Sakamoto H, Hayashida Y, Kuno M : Efferent discharges of sympathetic and parasympathetic nerve fibers during increased intracranial pressure in anesthetized cats in the absence and presence of pressor response. Brain Res 305 : 291-301, 1984
23. McBryde FD, Malpas SC, Paton JF : Intracranial mechanisms for preserving brain blood flow in health and disease. Acta Physiol (Oxf) 219 : 274-287, 2017

24. Münch $E$, Horn $P$, Schürer $L$, Piepgras $A$, Paul T, Schmiedek $P$ : Management of severe traumatic brain injury by decompressive craniectomy. Neurosurgery 47 : 315-322; discussion 322-323, 2000

25. Rangel-Castilla L, Gopinath S, Robertson CS : Management of intracranial hypertension. Neurol Clin 26 : 521-541, x, 2008

26. Reithmeier T, Speder B, Pakos P, Brinker G, Löhr M, Klug N, et al. : Delayed bilateral craniectomy for treatment of traumatic brain swelling in children: case report and review of the literature. Childs Nerv Syst 21 : 249-253; discussion 254, 2005

27. Robertson SC, Lennarson P, Hasan DM, Traynelis VC : Clinical course and surgical management of massive cerebral infarction. Neurosurgery 55 : 55-61; discussion 61-62, 2004

28. Ruf B, Heckmann M, Schroth I, Hügens-Penzel M, Reiss I, Borkhardt A, et al. : Early decompressive craniectomy and duraplasty for refractory intracranial hypertension in children: results of a pilot study. Crit Care 7 : R133-R138, 2003

29. Schmidt EA, Despas F, Pavy-Le Traon A, Czosnyka Z, Pickard JD, Rahmouni K, et al. : Intracranial pressure is a determinant of sympathetic activity. Front Physiol 9 : 11, 2018

30. Schwab S, Aschoff A, Spranger M, Albert F, Hacke W : The value of intracranial pressure monitoring in acute hemispheric stroke. Neurology 47 : 393-398, 1996

31. Shelton BA, O'Hara E, Tubbs RS, Shoja MM, Barker FG, Cohen-Gadol $A A$ : Emergency suboccipital decompression for respiratory arrest during supratentorial surgery: the untold story of a surgeon's courage in times of despair. J Neurosurg $110:$ 391-394, 2009

32. Smith ER, Carter BS, Ogilvy CS : Proposed use of prophylactic decompressive craniectomy in poor-grade aneurysmal subarachnoid hemorrhage patients presenting with associated large sylvian hematomas. Neurosurgery 51 : 117-124; discussion 124, 2002

33. Stefini R, Latronico N, Cornali C, Rasulo F, Bollati A : Emergent decompressive craniectomy in patients with fixed dilated pupils due to cerebral venous and dural sinus thrombosis: report of three cases. Neurosurgery 45 : 626-629; discussion 629-630, 1999

34. Stiefel MF, Heuer GG, Smith MJ, Bloom S, Maloney-Wilensky E, Gracias VH, et al. : Cerebral oxygenation following decompressive hemicraniectomy for the treatment of refractory intracranial hypertension. J Neurosurg $101: 241-247,2004$

35. Vahedi K, Vicaut E, Mateo J, Kurtz A, Orabi M, Guichard JP, et al. : Sequential-design, multicenter, randomized, controlled trial of early decompressive craniectomy in malignant middle cerebral artery infarction (DECIMAL trial). Stroke 38 : 2506-2517, 2007

36. Winter CD, Adamides A, Rosenfeld JV : The role of decompressive craniectomy in the management of traumatic brain injury: a critical review. J Clin Neurosci 12 : 619-623, 2005

37. Yoo DS, Kim DS, Cho KS, Huh PW, Park CK, Kang JK : Ventricular pressure monitoring during bilateral decompression with dural expansion. J Neurosurg 91 : 953-959, 1999 
BP and HR Changes during Decompression | Jo KW, et al.

38. Yumoto T, Mitsuhashi T, Yamakawa Y, lida A, Nosaka N, Tsukahara K, et al. : Impact of cushing's sign in the prehospital setting on predicting the need for immediate neurosurgical intervention in trauma patients: a na- tionwide retrospective observational study. Scand J Trauma Resusc

Emerg Med 24 : 147, 2016 